\title{
Inhibitory effects and mechanisms of several natural active components on inflammatory response of endothelial cell induced by activation of the complement alternative pathway
}

\author{
Qianyun Sun ${ }^{1,2}$, Min $\mathrm{Li}^{3}$, Jing Guo ${ }^{1,2}$, Jiao $\mathrm{Li}^{1,2}$, Fangjuan Zhu', Ying Zhou', Chaosheng $\mathrm{Li}^{1}$ \\ ${ }^{I}$ Center for Pharmacology and Bioactivity Research, The Key Laboratory of Chemistry for Natural Products of Guizhou \\ Province and Chinese Academy of Sciences, China, ${ }^{2}$ State Key Laboratory of Functions and Applications of Medicinal \\ Plants, Guizhou Medical University, China, ${ }^{3}$ General Ward, Guizhou Provincial People's Hospital, China
}

Background: Endothelial cell (EC) is an important multifunctional cell. Activation, dysfunction and injury of EC play an important role in a wide variety of diseases. Many reasons can induce activation, dysfunction and injury of EC, and the complement is highly involved in the initiation, amplification and effect of this process. Thus, the interest of this study is to investigate the potential inhibitory effect and mechanism of several natural active components on inflammatory response of endothelial cell induced by activation of the complement alternative pathway. Methods: The complement alternative pathway was specifically activated by cobra venom factor. The EC and mouse models of inflammatory response which was induced by the activated complement alternative pathway were employed to investigate the effects and mechanisms of chlorogenic acid, resveratrol, icariin, and quercetin on inhibiting the inflammatory response. Results: These active components inhibited the activation of NF-kappa B signaling pathway and up-regulation of transcriptional activity, and down-regulated the expression of related adhesion molecules and inflammatory mediators after EC was exposed to the components of the activated complement alternative pathway. The results in mice that treated with the active component revealed that inflammatory cell infiltration and myeloperoxidase activity in the lung tissue were significantly decreased. The cell count and content of protein, P-selectin, IL-6 and TNF alpha in bronchoalveolar lavage fluid were decreased, and IL-6, TNF alpha, P-selectin and ICAM-1 in serum were also decreased. Immunohistochemistry assay showed that phosphorylation of NF-kappa B p65 was inhibited. Conclusion: Chlorogenic acid, resveratrol, icariin, and quercetin can inhibit inflammatory response induced by activation of the complement alternative pathway, and the mechanism is highly related to the down-regulation of NF-kappa B activity and the decrease of inflammatory response. 